\title{
Microwave Assisted Synthesis of Some Quinoxaline Derivatives
}

\author{
AMIT DWIVEDI $^{* 1}$, ANSHU SINGH ${ }^{1}$ and ANIL MISHRA ${ }^{2}$ \\ ${ }^{1}$ Department of Chemistry, Dr. M C Saxena College of Engineering and Technology, \\ Lucknow, India \\ ${ }^{2}$ Department of Chemistry, University of Lucknow, Lucknow, India \\ amit_dwy@yahoo.co.in
}

Received 29 June 2013 / Accepted 10 July 2013

\begin{abstract}
The high commercial demand for quinoxalines needs a rapid, greener and safer synthetic method among the chemists. A series of quinoxaline derivatives has been synthesized by condensation of diamines and dicarboniles in microwave heating conditions and in solvent free media. This environmentally benign synthetic approach gives us excellent yields $(80-90 \%)$ in shorter reaction time (3.5 minutes.). The method is cleaner with an easier work-up process. The structures of these synthesized products are based on elemental analysis and mass spectral data.
\end{abstract}

Keywords: Green synthesis, Solvent free media, Microwave heating, Quinoxalines, Environmentally benign

\section{Introduction}

In the past few years, the use of microwaves in organic synthesis has increased dramatically, receiving widespread acceptance and becoming an indispensable tool ${ }^{1}$. Microwave technology to heat and drive chemical reactions has become a powerful tool in medicinal chemistry community, since by employing this technique it is generally possible to prepare organic compounds very fast, with high purity and better yields compared to other more conventional methods $\mathrm{s}^{2-4}$. One of the many advantages of using rapid 'microwave flash heating' for chemical synthesis is the surprised reduction in reaction times from days and hours to minutes and seconds $s^{5}$. The application of microwave heating under solvent-free conditions is promising alternative to polluting reaction and has been motivated the current field of interest.

Quinoxalines have extraordinary potential in pharmacological research ${ }^{6}$ and practice. These are important components of several pharmacologically active compounds ${ }^{7-13}$ and exhibit special and wider ranges of functions in biologically active compounds ${ }^{14}$ electroluminescent materials ${ }^{15}$, dyes ${ }^{16}$ and anion sensors ${ }^{17}$. Although rarely describe in nature, synthetic quinoxaline derivatives showed variety of pharmaceutical activities encompassed major types of drug target families and effective in many clinical applications such as anti tumor agents ${ }^{18}$, kinase inhibitors ${ }^{19}$, HIV drugs $^{20}$, antibiotics ${ }^{21}$, ion channel regulators $^{22}$ and anti protozoal agents ${ }^{23}$. 
Despite of various conventional methods described in literature for the synthesis of quinoxaline derivatives, synthesis of various new products embracing the principles of green chemistry $^{24,25}$ has gain new high in recent years. Microwave-assisted organic synthesis (MAOS), fuelled by the development and availability of precision controlled, single-mode microwave reactors, has had a profound impact on the way chemists approach organic and parallel synthesis. Clearly, reductions in reaction times, improved yields and suppression of side products, relative to traditional thermal heating, are benefits of this emerging technology ${ }^{26}$. Microwave-assisted protocols for the general synthesis of functionalized quinoxalines have been developed to provide rapid and high-yielding access to a variety of quinoxaline derivatives. In this publication the primary aim of our research was concerned on achieving reasonable yields of the synthesized heterocyclic products which might have biological and pharmaceutical prospective, using greener synthetic methodology. This work is inspired by Darabai et al. ${ }^{27}$ and this publication is an good extension to their work.

\section{Experimental}

Melting points were taken in an electrically heated instrument and are uncorrected. Compounds were routinely checked for their purity on silica gel TLC plates and the spots were visualized by Iodine vapors. PMR spectra were recorded on Buker DRX $300 \mathrm{MHz}$ FT NMR spectrometer using TMS as internal reference and chemical shift values are expressed in $\delta$ units. Mass spectra were run on Jeol SX-102 spectrometer.

\section{General synthetic procedure}

For the synthesis of these compounds, $0.4 \mathrm{~mL}$ of DMSO was added to a mixture of diamine $(1.1 \mathrm{mmol})$ and a dicarbonyl $(1 \mathrm{mmol})$ in an open glass tube and stirred for 2.5 minutes. The obtained $^{22-23}$ paste was exposed to microwave irradiations for 3.5 minutes in cycles of 9-10 seconds. The mixture was treated with water to dissolve the remaining amount of polar solvent. The precipitate was crystallized with hot aqueous ethanol. After cooling the filtrate, the resulted crystals were collected for filtration to afford the products ${ }^{1-8}$.

\section{Results and Discussion}

Microwave assisted chemical reactions have great potentials to be a 'green chemistry' tool, reduce environmental waste and use fewer chemical ingredients. The greener synthetic protocols were developed for synthesized quinoxaline derivatives. These products were easily synthesized in a shorter reaction time in comparison to the conventional methods. All the derivatives were obtained in very good amounts between $80-90 \%$ excellent yields. This process is safer, cleaner and environmental benign.

\section{Materials and methods}

The reaction mixture of diamine and dicarbonyl was mixed with proper amount of DMSO and exposed to microwave irradiations (Table 1). Using this greener and simpler synthetic procedures various quinoxaline derivatives as 2,3-dimethylquinoxalne, $6 \mathrm{H}$-indolo[2,3b]quinoxaline, 2,3-dimethyl-6-nitroquinoxaline, 3-nitro-6H-indolo[2,3-b]quinoxaline, $6 \mathrm{H}$ indolo[2,3-b] quinoxaline-3-carboxylic acid, quinoxaline-6-carboxylicacid, 6-nitroquinoxaline and 2,3-dimethylquinoxaline-6-carboxalineacid have been efficiently synthesized.
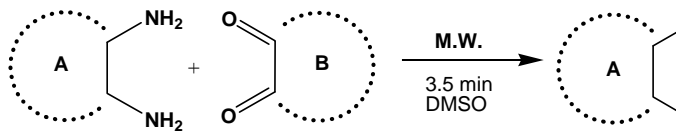

Scheme 1 
Table 1. Reaction details of quinoxaline derivatives

\begin{tabular}{|c|c|c|c|c|c|}
\hline Entry & Diamine & Dicarbonyl & $\begin{array}{l}\text { Molecular } \\
\text { formula } \\
\text { (Product) }\end{array}$ & $\begin{array}{c}\text { Yield } \\
\%\end{array}$ & $\underset{{ }^{0} \mathrm{C}}{\text { M.P. }}$ \\
\hline 1 & $o$-Phenylenediamine & 2,3-butadione & $\mathrm{C}_{10} \mathrm{H}_{10} \mathrm{~N}_{2}$ & 87 & 101 \\
\hline 2 & $o$-Phenylenediamine & Isatine & $\mathrm{C}_{14} \mathrm{H}_{9} \mathrm{~N}_{3}$ & 87 & 152 \\
\hline 3 & 4-Nitrobenzene-1,2-diamine & 2,3-butadione & $\mathrm{C}_{10} \mathrm{H}_{9} \mathrm{~N}_{2} \mathrm{O}_{2}$ & 90 & 151 \\
\hline 4 & 4-Nitrobenzene-1,2-diamine & Isatine & $\mathrm{C}_{14} \mathrm{H}_{8} \mathrm{~N}_{4} \mathrm{O}_{2}$ & 88 & $>250$ \\
\hline 5 & $\begin{array}{l}\text { 1,2-Phenylenediamine-4- } \\
\text { carboxylicacid }\end{array}$ & Isatine & $\mathrm{C}_{15} \mathrm{H}_{9} \mathrm{~N}_{3} \mathrm{O}_{2}$ & 80 & $>250$ \\
\hline 6 & $\begin{array}{l}\text { 1,2-Phenylenediamine-4- } \\
\text { carboxylicacid }\end{array}$ & Glyoxal & $\mathrm{C}_{9} \mathrm{H}_{6} \mathrm{~N}_{2} \mathrm{O}_{2}$ & 84 & $>250$ \\
\hline 7 & 4-Nitrobenzene-1,2-diamine & Glyoxal & $\mathrm{C}_{8} \mathrm{H}_{5} \mathrm{~N}_{3} \mathrm{O}_{2}$ & 90 & 192 \\
\hline 8 & $\begin{array}{l}\text { 1,2-Phenylenediamine-4- } \\
\text { carboxylicacid }\end{array}$ & 2,3-butadione & $\mathrm{C}_{11} \mathrm{H}_{10} \mathrm{~N}_{2} \mathrm{O}_{2}$ & 87 & 250 \\
\hline
\end{tabular}

Synthesis of 2,3-dimethylquinoxalne (1)

PMR (DMSOd $\left.)_{6}\right): 8.0(\mathrm{~d}, 2 \mathrm{H}, \mathrm{ArH}), 7.6(\mathrm{~d}, 2 \mathrm{H}, \mathrm{ArH}), 2.3\left(\mathrm{~s}, 6 \mathrm{H}, \mathrm{CH}_{3}\right), \mathrm{MS}(\mathrm{m} / \mathrm{e}): 158$ $\left(\mathrm{C}_{10} \mathrm{H}_{10} \mathrm{~N}_{2}\right), 130\left(\mathrm{C}_{8} \mathrm{H}_{6} \mathrm{~N}_{2}\right), 108\left(\mathrm{C}_{6} \mathrm{H}_{8} \mathrm{~N}_{2}\right), 80\left(\mathrm{C}_{4} \mathrm{H}_{4} \mathrm{~N}\right), 54\left(\mathrm{C}_{4} \mathrm{H}_{6}\right)$. Anal Cal. C, 75.92; H, $6.37 ; \mathrm{N}, 17.71$ Found $\mathrm{C}, 75.81 ; \mathrm{H}, 6.13 ; \mathrm{N}, 17.6443 .2$.

Synthesis of 6H-indolo[2,3-b] quinoxaline (2)

PMR (DMSOd $\left.{ }_{6}\right): 10.0(\mathrm{~s}, 1 \mathrm{H}, \mathrm{NH}) \quad 8.0(\mathrm{~d}, 2 \mathrm{H}, \mathrm{ArH}), 7.6(\mathrm{~d}, 2 \mathrm{H}, \mathrm{ArH}), 7.5(\mathrm{~d}, 1 \mathrm{H}, \mathrm{ArH})$, $\left.7.4(\mathrm{~d}, 1 \mathrm{H}, \mathrm{ArH}), 7.0(\mathrm{~d}, 2 \mathrm{H}, \mathrm{ArH}), \mathrm{MS}(\mathrm{m} / \mathrm{e}): 219\left(\mathrm{C}_{14} \mathrm{H}_{9} \mathrm{~N}_{3}\right), 169 \mathrm{C}_{10} \mathrm{H}_{7} \mathrm{~N}_{3}\right), 130\left(\mathrm{C}_{8} \mathrm{H}_{6} \mathrm{~N}_{2}\right)$, $107\left(\mathrm{C}_{6} \mathrm{H}_{6} \mathrm{~N}_{2}\right), 95\left(\mathrm{C}_{4} \mathrm{H}_{5} \mathrm{~N}_{2}\right), 119\left(\mathrm{C}_{8} \mathrm{H}_{9} \mathrm{~N}\right), 98\left(\mathrm{C}_{5} \mathrm{H}_{10} \mathrm{~N}\right), 80\left(\mathrm{C}_{4} \mathrm{H}_{9} \mathrm{~N}_{2}\right), 54\left(\mathrm{C}_{4} \mathrm{H}_{4}\right)$, Anal Cal. C, 76.70; H, 4.14; N, 19.17 Found C, 76.69; H, 4.09; N, 19.05.

\section{Synthesis of 2,3-dimethyl-6-nitroquinoxaline (3)}

PMR $\left(\mathrm{DMSOd}_{2}\right): 9.0(\mathrm{~d}, 3 \mathrm{H}, \mathrm{ArH}), 8.6(\mathrm{~d}, 1 \mathrm{H}, \mathrm{ArH}), 8.0(\mathrm{~d}, 1 \mathrm{H}, \mathrm{ArH}), 2.3(\mathrm{~s}, 3 \mathrm{H}$, $\left.\mathrm{CH}_{3}\right), \mathrm{MS}(\mathrm{m} / \mathrm{e}): 203\left(\mathrm{C}_{10} \mathrm{H}_{9} \mathrm{~N}_{3} \mathrm{O}_{2}\right), 189\left(\mathrm{C}_{9} \mathrm{H}_{7} \mathrm{~N}_{3} \mathrm{O}_{2}\right), 175\left(\mathrm{C}_{8} \mathrm{H}_{5}\right), 158\left(\mathrm{C}_{10} \mathrm{H}_{10} \mathrm{~N}_{2}\right), 153$ $\left(\mathrm{C}_{6} \mathrm{H}_{7} \mathrm{~N}_{2}\right), 130\left(\mathrm{C}_{8} \mathrm{H}_{6} \mathrm{~N}_{2}\right), 108\left(\mathrm{C}_{6} \mathrm{H}_{8} \mathrm{~N}_{2}\right), 99\left(\mathrm{C}_{4} \mathrm{H}_{5} \mathrm{NO}_{2}\right), 97\left(\mathrm{C}_{6} \mathrm{H}_{11} \mathrm{~N}\right), 80\left(\mathrm{C}_{4} \mathrm{H}_{4} \mathrm{~N}_{2}\right)$, $71\left(\mathrm{C}_{4} \mathrm{H}_{9} \mathrm{~N}\right)$, Anal Cal. C, 59.11; H, 4.46; N, 20.68; O, 15.75 Found C, 58.92; H, 4.38; N, 20.57; O, 15.68 .

\section{Synthesis of 3-Nitro-6H-indolo [2,3-b] quinoxaline (4)}

PMR (DMSOd $\left.{ }_{6}\right): 8.6(\mathrm{~d}, 1 \mathrm{H}, \operatorname{ArH}), 8.3(\mathrm{~d}, 1 \mathrm{H}, \operatorname{ArH}), 7.5(\mathrm{~d}, 1 \mathrm{H}, \operatorname{ArH}), 7.4(\mathrm{~d}, \mathrm{H}, \operatorname{ArH})$, $7.0(\mathrm{~d}, 2 \mathrm{H}, \mathrm{ArH}), \mathrm{MS}(\mathrm{m} / \mathrm{e}): 264\left(\mathrm{C}_{14} \mathrm{H}_{8} \mathrm{~N}_{4} \mathrm{O}_{2}\right), 219\left(\mathrm{C}_{14} \mathrm{H}_{9} \mathrm{~N}_{3}\right), 142\left(\mathrm{C}_{9} \mathrm{H}_{6} \mathrm{~N}_{2}\right), 130$ $\left(\mathrm{C}_{8} \mathrm{H}_{6} \mathrm{~N}_{2}\right), 108\left(\mathrm{C}_{8} \mathrm{H}_{8} \mathrm{~N}_{2}\right), 99\left(\mathrm{C}_{4} \mathrm{H}_{5} \mathrm{NO}_{2}\right), 98\left(\mathrm{C}_{5} \mathrm{H}_{10} \mathrm{~N}_{2}\right), 95\left(\mathrm{C}_{4} \mathrm{H}_{5} \mathrm{~N}_{3}\right), 80\left(\mathrm{C}_{4} \mathrm{H}_{4} \mathrm{~N}_{2}\right), 58$ $\left(\mathrm{C}_{2} \mathrm{H}_{6} \mathrm{~N}_{2}\right)$, Anal Cal. C, 63.64; H, 3.05; N, 21.20; O, 12.11 Found C, 63.70; H, 3.11; N, 21.18; O, 12.07 .

\section{Synthesis of 6H-Indolo[2,3-b]quinoxaline-3-carboxylic acid (5)}

PMR (DMSOd $)_{6}$ : 10.0 (s ,H, NH), 9.0 (d, 1H, ArH), 8.6 (d, 1H, ArH), 8.3 (d, 1H, ArH), 7.5 (d, 1H, ArH), 7.4 (d, 1H, ArH), 7.0 (bd, 2H, ArH), 6.8 (d, 1H, ArH 6.5 (d, 1H, ArH), 6.3 (d, $1 \mathrm{H}, \mathrm{ArH}), \mathrm{MS}(\mathrm{m} / \mathrm{e}): 263\left(\mathrm{C}_{15} \mathrm{H}_{9} \mathrm{~N}_{3} \mathrm{O}_{2}\right) 219\left(\mathrm{C}_{14} \mathrm{H}_{9} \mathrm{~N}_{3}\right), 130\left(\mathrm{C}_{8} \mathrm{H}_{6} \mathrm{~N}_{2}\right), 108\left(\mathrm{C}_{8} \mathrm{H}_{8} \mathrm{~N}_{2}\right), 98$ $\left(\mathrm{C}_{5} \mathrm{H}_{6} \mathrm{O}_{2}\right), 95\left(\mathrm{C}_{4} \mathrm{H}_{5} \mathrm{~N}_{3}\right), 80\left(\mathrm{C}_{4} \mathrm{H}_{4} \mathrm{~N}_{2}\right), 58\left(\mathrm{C}_{2} \mathrm{H}_{6} \mathrm{~N}_{2}\right)$. Anal Cal. C, 68.44; H, 3.45; N, 15.96; O, 12.16 Found. C, 68.49; H, 3.39; N, 15.90; O, 12.09 . 
Synthesis of Quinoxaline-6-carboxylicacid (6)

PMR (DMSOd 6 ): 8.9 (d, H, ArH), 8.7 (d, 2H, ArH), 8.5 (d, 1H, ArH), $8.2(\mathrm{~d}, 1 \mathrm{H}, \mathrm{ArH}), \mathrm{MS}$ $(\mathrm{m} / \mathrm{e}): 174\left(\mathrm{C}_{9} \mathrm{H}_{6} \mathrm{~N}_{2} \mathrm{O}_{2}\right), 152\left(\mathrm{C}_{7} \mathrm{H}_{8} \mathrm{~N}_{2} \mathrm{O}_{2}\right), 130\left(\mathrm{C}_{8} \mathrm{H}_{6} \mathrm{~N}_{2}\right), 127\left(\mathrm{C}_{6} \mathrm{H}_{9} \mathrm{NO}_{2}\right), 108\left(\mathrm{C}_{6} \mathrm{H}_{8} \mathrm{O}_{2}\right), 98$ $\left(\mathrm{C}_{5} \mathrm{H}_{10} \mathrm{~N}_{2}\right), 80\left(\mathrm{C}_{4} \mathrm{H}_{4} \mathrm{~N}\right), 56\left(\mathrm{C}_{2} \mathrm{H}_{4} \mathrm{~N}_{2}\right)$, Anal Cal. C, 62.07; H, 3.47; N, 16.09; O, 18.37 Found. C, 61.09; H, 3.40; N, 16.04; O, 18.40.

Synthesis of 6-Nitroquinoxaline (7)

PMR (DMSOd $\left.\left.{ }_{6}\right): 8.7(\mathrm{~d}, 2 \mathrm{H}, \operatorname{ArH}), 8.6(\mathrm{~d}, 1 \mathrm{H}, \operatorname{ArH}) 8.3(\mathrm{~d}, 1 \mathrm{H}, \operatorname{ArH})\right), \operatorname{MS}(\mathrm{m} / \mathrm{e}): 175$ $\left(\mathrm{C}_{8} \mathrm{H}_{5} \mathrm{~N}_{3} \mathrm{O}_{2}\right), 153\left(\mathrm{C}_{6} \mathrm{H}_{7} \mathrm{~N}_{3} \mathrm{O}_{2}\right), 130\left(\mathrm{C}_{8} \mathrm{H}_{6} \mathrm{~N}_{2}\right), 128\left(\mathrm{C}_{5} \mathrm{H} \mathrm{H}_{2} \mathrm{O}_{2}\right), 123\left(\mathrm{C}_{6} \mathrm{H}_{5} \mathrm{NO}_{2}\right), 99$ $\left(\mathrm{C}_{4} \mathrm{H}_{5} \mathrm{NO}_{2}\right), 80\left(\mathrm{C}_{4} \mathrm{H}_{4} \mathrm{~N}_{2}\right), 56\left(\mathrm{C}_{2} \mathrm{H}_{4} \mathrm{~N}_{2}\right)$, Anal Cal. C, 54.86; H, 2.88; N, 23.99; O, 18.27 Found. C, 54.65; H, 2.74; N, 23.76; O, 18.11.

\section{Synthesis of 2,3-Dimethylquinoxaline-6-carboxylicacid (8)}

PMR (DMSOd $\left.{ }_{6}\right): 8.9$ (d, 1H, ArH), $8.5(\mathrm{~d}, 1 \mathrm{H}, \mathrm{ArH}), 8.2(\mathrm{~d}, 1 \mathrm{H}, \mathrm{ArH}), 2.3\left(\mathrm{~s}, 3 \mathrm{H}, \mathrm{CH}_{3}\right)$, MS (m/e): $202\left(\mathrm{C}_{11} \mathrm{H}_{10} \mathrm{~N}_{2} \mathrm{O}_{2}\right), 188\left(\mathrm{C}_{10} \mathrm{H}_{8} \mathrm{~N}_{2} \mathrm{O}_{2}\right), 174\left(\mathrm{C}_{9} \mathrm{H}_{6} \mathrm{~N}_{2} \mathrm{O}_{2}\right), 158\left(\mathrm{C}_{10} \mathrm{H}_{10} \mathrm{~N}_{2}\right), 155$ $\left(\mathrm{C}_{8} \mathrm{H}_{13} \mathrm{NO}_{2}\right), 152\left(\mathrm{C}_{7} \mathrm{H}_{8} \mathrm{~N}_{2} \mathrm{O}_{2}\right), 130\left(\mathrm{C}_{8} \mathrm{H}_{6} \mathrm{~N}_{2}\right), 108\left(\mathrm{C}_{6} \mathrm{H}_{8} \mathrm{~N}_{2}\right), 98\left(\mathrm{C}_{5} \mathrm{H}_{6} \mathrm{O}_{2}\right), 80\left(\mathrm{C}_{4} \mathrm{H}_{4} \mathrm{~N}_{2}\right), 71$ $\left(\mathrm{C}_{4} \mathrm{H}_{9} \mathrm{~N}\right)$., Anal Cal. C, 65.34; H, 4.98; N, 13.85; O, 15.82 Found $\mathrm{C}, 65.29 ; \mathrm{H}, 5.03 ; \mathrm{N}$, $13.89 ; \mathrm{O}, 15.76$.

\section{Conclusion}

Different quinoxaline derivatives were successfully prepared using microwaves as source of heating following very easy work up process. This environment friendly procedure was accompanied by the reaction mixture of diamines and dicarbonyls with proper amount of DMSO which were exposed to microwave irradiations. All the products were found in excellent yields within a shorter reaction time. It is ample clear from these syntheses that a variety of quinoxaline derivatives may efficiently be prepared without using conventional methods.

\section{Acknowledgement}

The authors are thankful to the Director Central Drug Research Institute, Lucknow for providing $\mathrm{H}^{1} \mathrm{NMR}$ and mass spectroscopy. We are also thankful to the University Grant Commission, Delhi and University of Lucknow, Lucknow for the financial support.

\section{References}

1 Thierney J P and Lidstrm P, (Eds.), Microwave Assisted Organic Synthesis, Blackwell Publishing Ltd., 2005, 296.

2 Loupy A, Microwaves in Organic Synthesis, Wiley-VCH, Weinheim; 23 March 2004.

3 Hayes B L, Microwave Synthesis: Chemistry at the Speed of Light, CEM Publishing, Matthews NC, 2002.

4 Kappe C O and Stadler A, Microwaves in Organic and Medicinal Chemistry, WileyVCH, Weinheim, 2005.

$5 \quad$ Pecoraro E, Davolos M R and Jafelicci M J, Quim Nova., 1997, 20(1), 89-92; DOI:10.1590/S0100-40421997000100011.

6 Perumal R V and Mahesh R, Bioorg Med Chem Lett., 2006, 16(10), 2769-2772; DOI:10.1016/j.bmcl.2006.02.006.

7 Hassan S Y, Khattab S N, Bekhit A A and Amer A, Bioorg Med Chem Lett., 2006, 16(6), 1753-1756; DOI:10.1016/j.bmcl.2005.11.088. 
8 Zhao Z, Leister W H, Mahesh G D and Huff J R, Ronald G R, Stanley F B, Deborah D J, Raymond E J, George D H, Hans E H, Mark E D and Craig W L, Bioorg Med Chem Lett., 2005, 15(4), 905-909; DOI:10.1016/j.bmcl.2004.12.062.

9 Arther G, Elor K B, Robert G S, Guo Z Z, Richard J P, Stanley D, John R K, Sean T, Tammie J, Irene D, Steven M H, Bryan S M, Heath A McDonald, Prisca H, Carol T W, Kennan C M, Jill W, Kent D S, Tetsuro O, Michael F J, Carol S S, Connie R F and Chih H L, J Med Chem., 2005, 48(3), 744-752. DOI: 10.1021/jm0492958.

10 Andres J, Belen Z, Ibnacio A, Antonio M, J Med Chem., 2005, 48, 2019.

11 Ahmed A R, Mehta L K and Perric J, Tetrahendron, 1995, 51, 12899.

12 Sarges R, Howard H R, Browne R C, Label L A and Seymour P A, J Med Chem., 1990, 33, 2240.

13 Matsuoka M, Iwamato A and Furukawa N, J Heterocycl Chem., 1992, 29(2), 439-443; DOI:10.1002/jhet.5570290224.

14 Nasr M N A, Arch Pharma Med Chem., 2002, 8, 389-394.

15 Thomas K R J, Yu-Tai Tao and Chuen C H and Lin J T, J Master Chem., 2002, 12, 3516-3522; DOI: 10.1039/B206126K

16 Hirayama T, Yamasaki S, Hiroki A, Tsutomu Ishi-i, Thies $\mathrm{T}$ and Mataka S, Dyes Pigment., 2005, 67, 105-110; DOI:10.1016/j.dyepig.2004.09.023.

17 Ldakov D and Anzenbacher P, Chem Comm., 2003, 1339.

18 Torre M H, Gambino D, de Certain A 1, Jeannette Araujo, Hugo Cerecetto, Mercedes González, María Laura Lavaggi, Amaya Azqueta, Antonio Monge Vega, Ulrich Abram and Antonio J Costa-Filho, Euro J Med Chem., 2005, 40(5), 473-480; DOI:10.1016/j.ejmech.2004.11.012.

19 Gazit A, Yeek K and Uecker A, Bioorg Med Chem., 2003, 11(9), 2007-2018

20 Cheon H G, Lee C M, Kim B T and Hwang K J, Bioorg Med Chem Lett., 2004, 14, 1661.

21 Refaat H G, Moneeer A A and Khalil O M, Arch Pharma Res., 2004, 27, 1093-1098.

22 Bonde C, Norabeg J, Noer H and Zimmer J, Neurosci., 2005, 136(3), 779-794; DOI:10.1016/j.neuroscience.2005.07.020.

23 Hui X, Desrivot J, Borier C, Philippe M L, Xavier F, Reynald H and Bruno F, Bioorg Med Chem Lett., 2006, 16(4), 815-820; DOI:10.1016/j.bmcl.2005.11.025.

24 Anasta P T and Warner J C, Green Chemistry, Theory and Practice, Oxford University Press: New York, 1998, p.30.

25 Loupy A, Comptes Rendus Chimie, 2004, 7(2), 103-112; DOI:10.1016/j.crci.2003.10.015

26 Lidstrom P, Wathey B and Westman J, Tetrahedron, 2001, 57(45), 9225-9283; DOI:10.1016/S0040-4020(01)00906-1.

27 Mohsenzadeh F, Aghapoor K and Darabi R H, J Braz Chem Soc., 2007, 18(2), 297-303; DOI:10.1590/S0103-50532007000200009. 